\title{
ANAIS NIN O LA BÚSQUEDA DEL PADRE PERDIDO*
}

Juan Vives $R$.

Anaïs Nin nació en Neuilly, en las cercanías de Paris, el 25 de febrero de 1903. Era la hija mayor de Joaquín Nin, famoso pianista y compositor catalán, y de Rosa Culmell, cantante danesa criada y educada en La Habana, Cuba; tuvo la oportunidad de viajar por toda Europa durante su infancia, acompañando a su padre en sus triunfales giras, y de residir por temporadas en Francia, Bélgica, Alemania y la ciudad de Barcelona. Por ello es un tanto difícil encasillarla dentro de la literatura de algún país específico, y me parece preferible presentarla como una escritora franco-catalano-norteamericana, ya que tanto su personalidad como su obra están influidas primordialmente por las características de estos tres ámbitos culturales.

Conocemos el hecho de que, cuando la pequeña Anaïs contaba con once años y mientras residían en Barcelona, el padre, un conquistador impenitente, y enamorado hasta el delirio de una muchachita muy joven - Maruca-, decide romper con su familia, divorciarse, y modificar su existencia con su nueva y joven pareja. En estas condiciones, la madre de Anaïs decide tomar a sus tres hijos - su hija y dos varones menores - y embarcarse con rumbo a la ciudad de Nueva York, en donde tenía parientes, y en donde piensa refugiarse lejos de la espantosa humillación de la que se siente objeto por parte del que fue su marido.

Es así como Anais, luego de un primer período de su vida en el que difícilmente permanece sólida y consistentemente en algún lugar, se ve confrontada en la pre-pubertad a una nueva y más definitiva incertidumbre, al tener que hacer frente al rompimiento de sus padres, y a un cambio drástico, no sólo de país, sino incluso de continente.

En 1914 llega a los Estados Unidos, en donde hace sus estudios elementales, aprende una nueva lengua, se inicia en los pormenores de la profesión dramática al improvisar funciones de teatro para sus hermanos menores y otros niños del vecindario, se instruye y se interesa por el ancho mundo de la literatura, asistiendo a la biblioteca pública en la que comienza a leer las grandes obras de la literatura

* Trabajo leído durante el ciclo de conferencias "La recreación del creador", llevado a cabo en la Asociación Psicoanalítica Mexicana, México, D.F., noviembre de 1985. 
universal (cometido que realiza, a falta de mejor guia, por orden alfabético); en donde, posteriormente, se hace modelo profesional de pintores y fotógrafos; aprende ballet y baile español, actividades en las que logra ejecuciones más que buenas. Y, finalmente, en donde, además del estudio y del trabajo, se encarga de ser una auténtica segunda madre para sus dos hermanos menores, y un apoyo, guía y en ocasiones, principio de realidad para su deprimida progenitora.

Este período culmina alrededor de 1929, año en el que volvemos a encontrarla en el Viejo Continente, en el pueblito de Louveciennes, muy cercano a París, casada con un acaudalado banquero, e instalada en una bellísima finca que ella se encarga de convertir en un sitio encantador y oniroide.

Sin embargo, a Anals Nin la vida mullida la enmohece. Entendámosla en sus propias palabras:

Se vive asi, cobijado, en un mundo delicado, y uno cree que vive. Entonces lee un libro (Lady Chatterley, por ejemplo), o va de viaje, y descubre que no vive, que está simplemente hibernando. Los síntomas de la hibernación se pueden detectar fácilmente. El primero es la inquietud. El segundo síntoma es la ausencia de placer. Monotonía, aburrimiento, muerte. Hay millones de personas que viven (o mueren) así, sin saberlo (...) Sé que estoy en una bonita cárcel de la que sólo podré huir escribiendo. Por eso he escrito un libro sobre D.H. Lawrence, por gratitud, porque ese libro me despertó. (Diario, I, p. 17)*

Este despertar del adormilante letargo de mujercita inútil y acomodada en el que estaba sumida tuvo que ver, en lo esencial, con tres eventos trascendentales: en primer término, con su indeclinable pasión por la literatura, afición que siguió conservando pese a su cómodo encierro en Louveciennes; en segundo lugar, y derivado del anterior, por su descubrimiento de D.H. Lawrence, quien le enseña una nueva posibilidad para el mundo de la mujer, quien le abre otro tipo de espacio y le muestra la infinita posibilidad del ser femenino; $y$, finalmente, su providencial encuentro con Henry Miller, a quien conoce justo en el exacto momento en el que ya es capaz de atreverse a vivir en el mundo - al menos en ese mundo de Miller, en ocasiones un tanto brutal, pero pleno de sensaciones y de episodios insólitos; en suma, tan lleno de vida valiosa y significativa.

En este momento, el ensayo que Anals Nin dedica a D.H. Lawrence, y que se publica en 1932, es definitivamente un escrito revolucionario, sobre todo si tenemos en cuenta que nuestra autora es capaz de justipreciar y ensalzar la obra de un. hombre que, en esa época, era acusado de obsceno e incluso de pornográfico por toda la comunidad literaria europea.

En este sentido, no es de extrañar que ella misma también sea la "descubridora" de Henry Miller, otro consagrado de la literatura en lengua inglesa que, en sus

- Todas las citas al Diario de Anaïs Nin se refieren a la edición realizada por la Editorial $\mathbf{R}$ M, de Barcelona, excepto el tomo VI, en donde la referencia es al volumen publicado por la Editorial Bruguera. 
inicios, también fue señalado por el flamígero dedo de la moral burguesa, como pornográfico y destructor de los sacros valores tradicionales.

Sería largo, aunque muy sabroso, comentar la relación de Anaîs Nin con todo ese otro mundo de la literatura, paralelo al "gran" mundo que en esos momentos acaparaba la atención de la literatura norteamericana en París, mundo que se aglutinaba en torno a la figura de Gertrude Stein, y que, entre otros, incluía a gente como Hemingway y Scott Fitzgerald. El de Anaïs, sin embargo, tenía que ver con el mundo en penumbra de los "parientes pobres": gente como ese Miller, siempre pasando hambre y siempre "sableando" con un par de francos a quien se le pusiese cerca, ese Miller que estaba en París gracias a los esfuerzos infatigables de su bella esposa June, quien, beneficiándose de su trabajo como prostituta en Nueva York, había ahorrado dólar sobre dólar para enviar a su esposo al respirable ámbito literario del París de los años treinta.

Mucho más adelante, al final de la década, y en función de la estupidez humana concretada con èl nombre de Segunda Guerra Mundial, Anaïs Nin tendrá que emigrar nuevamente de París, también de nueva cuenta hacia los Estados Unidos, en donde residirá por el resto de su vida.

Pero, deteniéndonos un momento, lo que desearía enfatizar en este punto es el hecho de que, si bien es cierto que Anaiis Nin es indiscutiblemente la descubridora, promotora y estimuladora de la obra milleriana, también es verdad que Henry Miller, simultáneamente, descubre el talento de Anaïs Nin. Es Miller uno de los primeros en enterarse que la delicada mujercita de Louveciennes lleva muchísimos años escribiendo un Diario, al que tiene acceso a través de algunos de sus fragmentos, y bajo cuya impresión no tarda en declarar, sin ambages, que se trata de una obra de importancia tal que sólo es posible compararla con los más grandes documentos de ese género, cuando nos dice que el Diario de Anaiis "ocupará un lugar al lado de las revelaciones de San Agustín, Petronio, Abelardo, Rousseau y Proust".

Y este texto tan alabado por Miller, el famoso Diario, luegó de muchísimos años hará que el nombre de Anaïs Nin sea famoso en todo el mundo. La autora lo inició desde su remota infancia y, con el tiempo, llegó a ocupar más de 150 volúmenes manuscritos.

El inicio de este documento se remonta al año de 1914 , justo en el momento en que la pequeña Anaïs, de 11 años, se encuentra a bordo del vapor que la lleva, con su madre y sus dos hermanos menores, a los Estados Unidos, luego del traumático divorcio de sus padres. En este momento el Diario comienza en forma de una carta que la pequeña escribe a su padre, epistola que tiene como finalidad consciente la intención de convencer al padre de que regrese con su familia; es decir, que tiene como motor la necesidad de re-conquistarlo (y decimos re-conquistarlo porque como veremos más adelante, el padre ya había sido conquistado en años previos por la seductora Anaïs). Ante el indescriptible dolor de la separación, la carta -luego continuada como Diario - tiene como objetivo el intentar convencer al padre de que se reúna con ellos y no los abandone.

Muchos años después, Anaïs será plenaménte consciente de que algo muy serio 
sucedió dentro de ella cuando sus padres se divorciaron; nos dice: “...alguna cosa en la psique fue destruida ... después del abandono de mi padre, algo que impidió mi desarrollo, mi seguridad y mi madurez" (Diario, IV, p. 186).

En otros términos, desde su mero inicio el Diario emerge desde el signo de una ausencia -la del padre - y tiene una finalidad: re-establecer el vínculo perdido. Y si bien esta intención inicial se va modificando con el tiempo, el Diario conservará siempre, sin embargo, ese atributo de vínculo, de diálogo con un objeto ausente. A la vez, poco a poco, irá tomando insensiblemente la forma de una gran obra literaria. De esta manera, Anaïs Nin llegará a ser una escritora de prestigio literario a pesar de ser una autora de novelas relativamente poco conocidas, como Hijos de Albatros, Corazón cuarteado, La seducción del Minotauro, y otras; obras que de hecho han salido a la luz y han sido conocidas por el gran público sólo con posterioridad a la publicación del Diario. Incluso, como novelista, Anaïs Nin es más conocida por su obras relativamente menores como Delta de Venus y Pajaros de fuego, que son obras eróticas de escaso valor literario, que por sus obras de mayor envergadura como Escaleras de fuego o Bajo una campana de cristal.

Pero el Diario, que como se dijo anteriormente sobrepasaba los 150 volúmenes en el momento de morir su autora (en 1977), ha hecho de ella una gran figura de la narrativa contemporánea. Lamentablemente, los seis volúmenes que se publicaron constituyen una rigurosa selección del material original, dado que contenía tal cantidad to datos y observaciones en torno a conocidas personalidades tanto del mundo del arte en general y de la literatura en particular, como de la política y del mundillo social. Esas personas - muchas de ellas vivas aún - hacían imposible su edición sin antes ejercer una enérgica censura, operación que llevó a cabo la propia autora y que, en consecuencia, transformó en cierta manera aquel documento original, espontáneo y veraz, en un producto depurado, estéticamente más sofisticado, pero psicológicamente castrado. La mutilación incluye, como parte muy importante, el silencio de la autora en torno a las relaciones con su marido, así como otras relaciones, amores y amoríos, y el relato pormenorizado de sus vivencias de lo erótico y de su sexualidad, experiencias que tuvieron que ser acalladas por completo. En esta forma, este Diario ad usum delphini que la autora nos ha legado, constituye una suerte de selección apretada, aunque conserva, sin embargo, gran parte de su valor intrínseco al informarnos de lo más esencial de la vida de su autora, y de sus relaciones con el mundo del arte y de la creación; relato que sí nos permite adentrarnos en algunos de los factores internos que tienen que ver con el proceso de gestación del acto creativo, o por lo menos vislumbrarlos.

Ahora bien, el Diario - esta segunda versión pulida y abreviada - nos ofrece la posibilidad de investigación en múltiples líneas, tantas que uno podría fácilmente dispersarse en función de la riqueza del material ofrecido o en la seducción de los cientos de anécdotas significativas contenidas entre sus densas páginas. En estas circunstancias, es importante poder renunciar a varias de las líneas de investigación posibles, para ceñirnos a una sola de ellas, que a la manera de hilo rojo nos guíe en la lectura a través de los varios volúmenes de tan extensa crónica, y que puede ser el seguimiento de una de las primerísimas intenciones de su autora cuando se puso a 
escribirlo por primera vez, allá en la lejanía de sus once años, ante el hueco dejado por su padre.

Además, esta línea de investigación tiene la ventaja de que nos lleva de lleno al estudio de la estructura de la personalidad de Anaiis Nin, con las disociaciones que resultaron de las vicisitudes de sus relaciones objetales, así como al análisis de los cimientos de su identidad, derivados de los diversos procesos de identificación en el curso de su azarosa vida, tanto con su madre como con su padre.

Al perder al padre, Anais no sólo hace de él un ser idealizado y mítico, sino que se identifica con él, ya que al incorporarlo dentro de sí misma tiene la ilusión de evitar - y negar - su pérdida. Es por ello que, para Anaïs, su identidad como artista tiene que ver con una forma de conservar al padre dentro de ella. Al mismo tiempo, su pérdida tiene un significado particular, que, en su fantasía, podría ser expresado con los siguientes pensamientos: si su padre la ha abandonado, seguramente se ha debido al hecho de que ella no se ha portado bien; es decir, que la han abandonado porque ella ha sido mala. Debido a esta psicodinamia, y desde aquellos sus remotos once años, Anaîs conservará la huella del efecto traumático de esta fantasía y de esta herida narcisista, ya que a partir de entonces impone una profunda disociación a su personalidad, cuyo resultado se manifiesta en una incapacidad para expresar cualquier tipo de conducta agresiva: le es imposible experimentar la vivencia del odio $y$, consecuentemente, se sentencia a proyectar estos elementos siempre en los demás.

Al mismo tiempo que ocurren estos sucesos en el vínculo con su padre, el proceso de identificación con su madre se ve alterado en ciertos aspectos, ya que esta última le representa a la tradición española católica, asfixiante y castrante, mediocre y fracasada: doña Rosa había renunciado a la cantante que pudo haber sido, al decidirse a ser un simple satélite de su inteligente y agraciado esposo.

En esta forma, al identificarse primordialmente con la figura del padre, Anais repudia la pasividad femenina de su madre, y abraza la posibilidad del arte, del artista que significa su padre, que para ella representa la sensibilidad exquisita, el buen gusto, la cultura refinada, los valores más sofisticados de nuestra civilización. Pero al mismo tiempo, el padre también le significa la figura narcisista por excelencia, el hombre que nunca pudo establecer una relación de amor con nadie, ni siquiera con su propia hija.

En este sentido, Anaïs Nin queda atrapada entre dos figuras de identificación, hecho que determina que durante casi toda su vida - al menos en lo aparenterechace a la figura de su madre y construya su existencia alrededor de la búsqueda de ese padre perdido, ausente e idealizado. Y este quedar atrapada representará el mayor punto de fijación de Anais, punto que determinará que, en estricto sentido, la autora de La casa del incesto intente en la realidad, y más allá de la apariencia de búsqueda del padre mítico, el encuentro con lo que nunca pudo obtener de su madre, es decir, el mundo del afecto, de la seguridad, de la confianza, de una madre que le construyera la imagen de un padre bueno y real, y un espacio de respirable respeto en el que ella pudiera desarrollarse en su derecho propio. Así, el Diario, a la vez que intenta recuperar el vínculo con el padre, es un mecanismo mágico que la 
protege contra las pérdidas. Ella sabe que las experiencias de la vida pasan, que son efímeras, y que nuestra única oportunidad de sentir su continuidad reside en nuestra memoria. Si ella registra sus experiencias en un Diario, se asegura de que dichas vivencias no se pierdan jamás: "Seguramente esta necesidad de registrar inmediatamente es lo que me impulsa a escribir casi al mismo tiempo que vivo, antes de que cambie, antes de que la distancia o el tiempo me alteren"(Diario, I, p. 73).

Como vemos, nuevamente aparece el problema del bloqueo de la agresión, a través de este temor a la más definitiva de las pérdidas: la muerte.

Pero volviendo al interjuego de las identificaciones, podemos ver cómo el matrimonio que hace Anaiis con un banquero, más allá de su apariencia de reencuentro con un padre fuerte y sólido, es en realidad la realización del deseo de una madre consistente y segura. De hecho, su madre fue tan ineficaz y pusilánime en el mantenimiento de su familia que Anaïs, desde muy joven, no sólo tuvo que ejercer funciones maternales con sus hermanos menores, sino que, incluso, apenas tuvo 16 años, se vio en la necesidad de salir, casi de emergencia, de su casa, para trabajar en la Asociación de Modelos de Nueva York, posando para pintores y fotógrafos, y hacerse cargo de las finanzas de su familia.

Por cierto que no es casual la elección de ésta, su primera profesión, que tiene tanto que ver con la posterior estructuración narcisista de la personalidad de Anaïs Nin, ya que representa, simbólicamente, el único vínculo posible con el padre -vínculo sustentado en la pasión fotográfica de su progenitor-, a la vez que constituye su muy efectiva manera de seducirlo y tenerlo conquistado para sí en el período edípico. Dice Anaïs: “Le gustaba hacerme fotos cuando me bañaba. Quería que siempre estuviera desnuda. Toda su admiración se canalizaba a través de la cámara" (Diario, I, p. 95).

De esta suerte, no es extraño que luego Anaïs se haya dedicado a posar para fotógrafos y pintores, y que haya basado tantas de sus relaciones en su personal capacidad de seducción; no es raro que, en el curso de su primer psicoanálisis, en vez de describir sus pechos, haya preferido, lisa y llanamente, despojarse de la blusa y mostrárselos directamente a su analista; no es de extrañar que hasta bien avanzada su vida haya tenido pasión por ser fotografiada y que, sin ser actriz, haya aparecido en varias películas. No causa sorpresa, finalmente, que Anaïs Nin haya pasado a la posteridad por su Diario, que es una forma artística y literaria de mostrarnos hasta los más recónditos rincones de su intimidad.

Por otro lado, debemos tener en cuenta que parte muy fundamental de dicha estructuración narcisista se vio facilitada por la extraordinaria belleza de Anaïs Nin, desde jovencita; belleza que no sólo estaba basada en estrictos atributos físicos, sino primordialmente en el hecho de que era una mujer que ejercía una inquietante fascinación sobre la gente, hombres y mujeres, gracias a que expresaba una intensa femineidad. Ese tipo particular de femineidad un tanto frágil en lo aparente, que provocaba una gran atracción sobre los hombres, para los cuales Anaïs representaba una suerte de mujer-niña-ángel, incluso un tanto desexualizada -en el sentido de que la autora de Un espía en la casa del amor no era una mujer 
abiertamente sensual, aunque ejercía un tipo aún más intenso de fascinación a través de otra clase de atributos.

Desde el punto de vista de esta caracterologia narcisista, el Diario de Anaï Nin constituye una especie de espejo: es como aquellas aguas del río en las que Narciso se mira y admira. En esta forma vemos cómo este documento, además de su utilidad como vínculo simbólico con la figura del padre, se constituye al mismo tiempo en un doble (en el sentido rankiano) de la propia Anaïs, por cierto su doble más sincero.

$\mathrm{Y}$ aquí es donde las consideraciones anteriores en torno al fenómeno del doble desembocan en la develación de esa otra gran característica de Anaïs Nin: su personalidad tipo "como si". Nuestra heroína es, desde su más remota infancia, en la que preparaba funciones de teatro en la cocina de su casa para entretener a sus hermanos, una inveterada actuadora de papeles: Anaïs llega a especializarse en ser una actriz de la vida, con la particularidad de que tiene plena conciencia de que constantemente actúa papeles; advertencia trágica y dolorosa, ya que tiene la clara vivencia de estar enejanada de sí misma, de poseer múltiples yoes disociados los unos de los otros. Dice textualmente:

En mi vida han existido siempre dos mujeres al menos, una desesperada y perpleja, que tiene la sensación de estar ahogándose, y otra que sólo quiere dar belleza, gracia y vida a la gente, y que entra en escena, como si fuera en un escenario, ocultando sus verdaderas emociones porque son debilidades, desesperación, desconsuelo, y presentando al mundo solamente una sonrisa, entusiasmo, curiosidad, interés. (Diario, I, p. 281)

Pero además de su doliente conciencia de fragmentación interna en varios yoes disociados, Anaïs, con su conocimiento de la vida y de las personas, nos advierte que este fenómeno que a ella le ocurre es una experiencia vista con la lente de aumento de su capacidad introspectiva, ya que, en forma más o menos encubierta, toda la gente posee múltiples yoes, sólo que la mayoría de las personas reprimen o sepultan en su inconsciente todas estas otras partes disociadas, fragmentos que la autora no sólo asume como propios, sino que los actualiza, los potencia y los actúa hasta sus últimas consecuencias. Por eso nos dice: "Hay tantas cosas dentro de cada uno de nosotros, cosas en potencia, inconscientes, que si tienes que interpretar un papel, y ese papel corresponde al Yo dormido, este yo despierta, se hace realidad" (Diario, Il, p. 176).

Para Anaìs, como es obvio, la plena realización de la personalidad reside en la posibilidad de integrar los múltiples fragmentos de los que el Yo está compuesto, integrarlos y vivirlos: atreverse a experimentar cada una de esas personalidades, cada una de esas facetas a través de las cuales se manifiesta la existencia toda. De lo contrario ... "Vivir solamente un aspecto, o un lado, de la personalidad es como utilizar sólo un sentido: los otros se atrofian" (Diario, II, p. 248).

Quizás uno de los aspectos más emocionantes para el lector del Diario es la oportunidad que su autora nos brinda para asistir, a través de sus seis dilatados 
volúmenes, al proceso del desarrollo, avatares, tropiezos y esfuerzos de esta personalidad "como si", en sus múltiples actuaciones, frecuentemente contradictorias y antitéticas, en su afanosa lucha, no sólo por la integración de sus muchos fragmentos, sino por la plena realización de cada uno de ellos, que, en esta forma, lentamente, van tomando cuerpo, cada vez más real y genuino: cada vez menos actuación y cada vez más acción verdadera -en el desarrollo y descubrimiento de su auténtico ser.

Al final, asistimos a una Anaïs integrada, participamos en la lectura de una persona (ya no de un personaje) a la que, ahora sí, visualizamos; a la que oímos respirar, reír, gozar y llorar. Anteriormente, durante el recorrido de los primeros volúmenes, uno tenía la desagradable sensación de estar asistiendo siempre a la representación de uno de sus personajes, a una sucesión interminable de disfraces detrás de los cuales la autora siempre se cuidaba de que quedara bien oculta la pequeña niña Anaïs, solitaria, temerosa y hambrienta. Esa niña que no pudo crecer siguiendo sus naturales inclinaciones. Ya bien adelantada en su vida, y con la sabiduría que le proporciona la distancia de los hechos y la experiencia vivida, nos dice la autora: "Cuando somos niños, nos hacen ver que sólo nos quieren cuando nos portamos bien (en términos paternos). Desde el momento en que empezamos a mostrar nuestro Yo real, los padres comienzan a rechazarnos. Crecemos con la idea de que si somos nosotros mismos, nos rechazarán" (Diario IV, p. 101).

Con una perspicacia poco común, Anaïs Nin nos enseña el proceso mediante el cual un niño - una niña en este caso -, en vez de erigir un saludable Yo, una fuerte y flexible sensación de mismidad, tiene que doblegarse y "fingir" aquel Yo (falso), aquella identidad que los padres quieren para ellos, fraude gracias al cual la pequeña obtiene lo que con mayor desesperación necesita: el cariño y la aceptación de sus progenitores. El precio, entre tanto, es crecer con un falso self, con un Yo abortado de sus auténticas posibilidades, una existencia enajenada del cuerpo en el que se asienta, disociada de la piel que la contiene y la define.

De cualquier modo, en justicia el Diario siempre conservará la característica de ser la parte más genuina, más verdadera, de Anaiis Nin; incluso podríamos decir también que su parte más creativa. No es casual que frente al escaso valor de su novelística, basada en personajes, el Diario, basado en la persona, sea un auténtico documento literario de valor indiscutible. El Diario es ese espacio en el que Anaïs, luego de la larga jornada de actriz de la vida, puede quitarse sus ropajes de utileria y, finalmente, en ese tiempo privado para el recogimiento que ella tanto aprecia, ser ella misma: relajada, pausadamente, la autora recuerda, re-crea, re-vivencia a cada uno de sus personajes, observándolos ahora desde su genuina persona. Observa las evoluciones de sus dobles, anota sus características, describe y descubre sus trucos. El Diario, ese doble especular, se constituye así en su más íntimo confidente, casi en una suerte de compañero imaginario con el que dialoga fantásticamente; a veces también, sus escritos constituyen su única y melancólica compañía. Al único al que puede abrirse y, desnuda, confesarle:

Mi vida no ha sido sino un prolongado esfuerzo por crear, por convertirme en alguien interesante, por desarrollar mis dotes, por hacer que mi padre estuviese 
orgulloso de mí, una desesperada y angustiosa ascensión por borrar y destruir un obsesivo sentimiento de inseguridad, fruto de la convicción de que mi padre nos dejó porque yo le decepcioné, porque no me quería; y porque la mujer a quien amaba era Maruca. He apuntado cada vez más alto, acumulando amores en compensación del primero que perdi. Amores, libros, creaciones. (Diario, I, p. 213)

Es como si Anaïs Nin nunca hubiese podido remontar del todo la experiencia de aquella pequeña que se quedó gritando de dolor y de terror al sentirse abandonada por su padre. Su narcisismo, es decir, su necesidad de sentirse centro y causa de aquel evento, es lo que la atrapa en la red mortal de su sensación de maldad, de que su perversidad fue la causante del abandono, y en el sentimiento de culpa resultante.

Gracias a factores constitucionales que inmediatamente analizaremos, esta experiencia no fue devastadora; sin embargo, para nosotros es evidente que aquel suceso infantil constituyó algo muy parecido a una quiebra psicótica, luego de la cual Anaiis, en vez de hacer una re-conexión delirante con el mundo, como hubiese hecho cualquier psicótico, hizo en cambio una re-conexión desde la creatividad, desde una posición de re-creación. Ella misma nos lo dice: "Me parece que toda mi creación es un esfuerzo por tejer una red de conexiones con el mundo; estoy constantemente tejiéndola porque hubo una vez que se rompió" (Diario, III, p. 244).

La autora de Corazón cuarteado es una profunda e implacable conocedora de sí misma, por eso nos puede decir: "Yo sé cuáles son mis crímenes. Lo que otros seres humanos sólo sueñan yo lo realicé. Pero soy incapaz de liberarme del sentimiento de culpa" (Diario, VI, p. 48).

Es la culpa primigenia por realizar los propios deseos, tan patentes y transparentes en los sueños; es la culpa por llegar a ser ella misma, independientemente de los deseos de papá y de mamá; es la culpa original (el gran pecado) consistente en existir, ser y conocer:

Nadie puede hacer lo que yo he hecho, lo sé, porque fue precisa una visión espiritual aliada con la sensualidad para poder revestir de carne significados tan profundos, y fue necesaria una vida en el infierno y muchas vidas de penosas exploraciones, e incluso una peligrosa estancia en el mundo de la locura, y la capacidad de volver para contar lo que he contado (Diario, V, p. 87)

Pero ¿qué fue lo que determinó que una experiencia de características tales, promotora con toda seguridad de algo muy parecido a una quiebra psicótica, no ejerciera efectos devastadores sobre Anaïs Nin? Para mí, en este punto tenemos que invocar la presencia de una serie de factores de índole constitucional, con el fin de poder intentar una explicación plausible. Partiendo desde lo más evidente, es claro que Anaïs, desde muy pequeña, posee una hipersensibilidad exquisita para la captación del mundo circundante, incluidos sus propios procesos de pensamiento; característica que, unida a su capacidad para expresar estos contenidos a través de un lenguaje simbólico pleno de cualidades estéticas, hace de ella una artista nata y 
una personalidad creadora. Pero más allá de estos factores, lo que nos desconcierta y admira en Anaïs Nin es su peculiar forma de re-conectarse con el mundo. En vez de una re-conexión delirante, y por lo tanto definitivamente apartada de la realidad objetiva, en vez de una re-conexión cotidiana, simplemente vinculándose con objetos reales tal y como están en el mundo, Anaïs hace una re-conexión intermedia entre la fantasía y la realidad, vinculación que conserva, milagrosamente, las características de a mbos mundos, idéntica al juego alucinatorio del niño, mediante el cual el campo de batalla en el que trascurren sus aventuras, simultáneamente está lleno de tanques, soldados y cañones, y es un pedazo de jardín lleno de informes trozos de madera y de cacharros de la vajilla de mamá. Esta característica tan clara en el niño, mediante la cual un pedazo de madera es realmente un tanque de guerra que dispara balas y mata soldados, y a la vez también es realmente sólo un pedazo de madera, es una característica constante en multitud de creadores, que los hace aparecer un tanto niños o cándidos ante los ojos de sus contemporáneos.

Anaïs Nin nos lo dice con muy pocas palabras, pero plenas de sentido: “...se ha dado por fin una fusión de la vida con la fantasía, del sueño con la acción, que ahora forman una sola corriente. Sueño, realizo el sueño; vivo, sueño lo que vivo, y esto alimenta el siguiente paso, que es la narración" (Diario, III, p. 108).

En la actividad creativa que se da a través de la narración, podemos asistir al proceso interno mediante el cual el creador, ante un mundo vivido como frustrante y miserable, crea (o mejor dicho, re-crea) -en una identificación con Dios, y más primariamente, con las primeras deidades femeninas genésicas - un nuevo mundo sin sobresaltos, un mundo sin frustraciones, amable, confortable, en donde las cosas suceden a la medida de los deseos, de las necesidades, del color de nuestros sueños. Un mundo que, gracias a sus cualidades estéticas formales de armonía, ritmo y equilibrio, el autor comparte con los espectadores de su obra, y al hacerlo, no sólo les proporciona un mensaje valorado como valioso desde el punto de vista de su belleza inherente, sino que les proporciona la oportunidad de escapar, ellos también, y aunque sea momentánea y vicariamente, de las penurias y miserias reales del mundo objetivo, con todas sus presiones, malestares y condicionamientos.

Desde este punto de vista Anaïs Nin es la típica personalidad creadora, poseedora de una enorme fuerza yoica, de una capacidad regresiva notable y de una hipersensibilidad fuera de lo común para la captación de hasta los más mínimos detalles del mundo circundante, y de ella misma. Por ello Anaïs ha podido llegar a hacer de la introspección un auténtico arte, tal como se manifiesta en cada una de las páginas de su Diario.

Al mismo tiempo, en Anais Nin puede apreciarse, como en pocas personas, cómo su creatividad y su arte tienen que ver con sus partes integradas y no con su patología disociativa. Su arte tiene relación con el Diario, es decir, con su yo más integrado y profundo; en cambio, sus novelas obedecen más a la reseña de sus personajes escindidos, falsos, orbitales. Su patología la fragmentó, la enloqueció, la hizo una inválida durante buena parte de su existencia. Como ella dice: "La primera persona a la que me había entregado, mi padre, me traicionó, hasta que exploté. Es fatal entregarse completa mente. Reviento, me subdivido en un millón de relaciones 
pequeñas. Y busco a seres reventados, a los seres subdivididos"(Diario, IV, p. 140).

Su patología, al disociarla, la hizo sentir y pensar de esta manera, y mucho. tiempo habría de costarle (y el paso por cuatro experiencias psicoanalíticas, con Allendy, Rank, Jaeger y Bogner) el poder recuperar su capacidad de entrega total, el renunciar a esa multifragmentación en cientos de amigos, pseudorrelaciones que la protegían de la posibilidad de un auténtico involucramiento afectivo.

Por el contrario, las fuerzas de su Yo, sus capacidades integrativas hicieron de ella no sólo la creadora de una gran obra literaria, sino que posibilitaron sus capacidades de reparación, al poder admitir dentro de ella, finalmente, la rabia, ila temida rabia!, que en su fantasía había provocado esa explosión de la que constantemente habla.

Así, en el curso de su tercer análisis nos confiesa: “A [la Dra.] Jaeger le preocupa mi esquivez, todo lo he puesto fuera de mí, en los demás. No soportaba pensar que había en mí algún elemento destructivo" (Diario, III, p. 308). Y mucho más adelante, ya en su cuarta experiencia psicoanalítica, agrega: "He de escaparme cuando me siento ofendida porque tengo miedo de $\mathrm{mi}$ ira. El verdadero problema está en qué debo hacer con mi ira. La he sofocado durante tanto tiempo que está cargada como la dinamita, la he almacenado y a hora amenaza con salir en forma de explosiones que me dan miedo" (Diario, VI, p. 140).

Esto explica otra de las características más constantes en la autora de Hijos de Albatros: que a pesar de ser, en lo aparente, una eterna buscadora de un padre, compulsión que determina buena parte de sus relaciones con los hombres, desde su marido el banquero hasta Henry Miller, Lawrence Durrell, Gonzalo, R. Allendy, O. Rank, y otros, en la práctica siempre se las arregla para terminar siendo ella quien los protege a ellos. Y esta última característica, aparentemente tan maternal en Anaïs, es en realidad un camuflaje, ya que en su mundo interno lo que hace es proteger a todos esos hombres, pero de su propia agresión, la que ella siente tan amenazante, la que vive con tanto terror.

Esto explica una curiosa anécd ota en la vida de Anaïs Nin: alguien le pregunta:

- ¿Está usted muy interesada por Lawrence?

-Ahora ya no tanto. Ya no necesita que nadie lo defienda (Diario, I, p. 187).

Como podemos verlo con claridad, tanto D.H. Lawrence,ese autor "obsceno y pornográfico" que fue objeto de su primer estudio y al que defendió con ardor, como luego Henry Miller, y luego incluso algunos psicoanalistas, simbólicamente eran representantes de ese padre libertino, donjuanesco y sensual, al que la niña Anaïs ama, pero que al mismo tiempo, y ante el abandono, es objeto de todos sus rabiosos ataques; agresiones fantaseadas de las que ella misma, a través de la protección que ejerce sobre los subrogados paternos, lo protege y lo cuida, como una manera de no agudizar su dolorosa orfandad.

Son las fuerzas integradoras en el Yo de Anaïs Nin las que nos permiten asistir, como emocionados lectores, a su proceso de convertirse en persona. Recuperada e integrada dentro de ella su capacidad de odio, ahora sí ya puede actualizar también su capacidad de amor. En este momento, la mujer creadora que ha madurado ya puede incluso renunciar a la gratificación narcisista de ser el centro maternal de 
toda una corte de aduladores, niñitos voraces que la explotaban inmisericordemente. Recuperada su autoestima, puede también comenzar a gratificarse con las pequeñas cosas que toda existencia ofrece.

Más allá de las etiquetas psicopatológicas que seguramente podemos aplicarle a nuestra biografiada, por coherentes que éstas sean, y más allá de todas las consideraciones psicodinámicas que con justicia y verdad podemos elaborar en torno a su obra y su vida, nos queda el escueto hecho de la presencia de una mujer cuya principal creación, y a la vez la más ardua y fatigosa, fue su propia vida; la presencia de una mujer vital, alada, que hizo de su mera existencia lo más maravilloso que con ella puede hacerse: algo digno de ser vivido.

\section{BIBLIOGRAFIA}

NIN, A. Diario I (1931-1934). Trad. de E. Hegewicz, Barcelona, Editorial R M, 1977.

- - Diario II (1934-1939). Trad. de E. Hegewicz, Barcelona, Editorial R M, 1978.

- - Diario III (1939-1944). Trad. de E. Hegewicz, Barcelona, Editorial R M, 1979.

-_- Diario IV (1944-1947). Trad. de E. Hegewicz, Barcelona, Editorial R M, 1979.

- - Diario V(1947-1955). Trad. de E. Champourcin y A. Tudela, Barcelona, Editorial R M, 1980.

- - Diario VI (1955-1966). Trad. de L. Graves, Barcelona, Editorial Bruguera, 1984. 